\title{
SIMPLE PLASTIC PROCEDURES IN GENERAL SURGERY
}

\author{
By F. T. MOoRE, F.R.C.S., F.R.C.S.E. \\ Plastic Surgeon, King's College Hospital, London; Surgeon, Plastic Unit, East Grinstead
}

Plastic surgery deals with the restoration of parts of the body destroyed or deformed as the result of congenital defect, trauma, surgical excision or disease. It should have for its main object the restoration of function. It should also be the aim of the surgeon to secure a good cosmetic result. In all cases the principle of restoration of tissue in kind-bone for bone and skin for skinis of paramount importance.

It is the purpose of this paper to describe simple procedures used by plastic surgeons and within the scope of all general surgeons.

Plastic surgeons have developed a technique which can rightly be called atraumatic. It is quite impossible not to inflict damage to tissues if unsuitable instruments are used. Many general surgical instruments such as haemostats, tissue forceps, retractors, needles and suture material are much too heavy and deny any feeling or touch to the surgeon. The greatest care possible should be taken when it is necessary to handle tissues. Maingot has taught for years that further advances in abdominal surgery will come from the free adaption of plastic technique to abdominal operations.

\section{Closure of a Skin Incision}

It is pertinent at this point to describe the method used by plastic surgeons to complete closure of a skin incision. The instruments used are lightweight forceps of the McIndoe type, dural hooks to retract the skin, the mosquito pattern artery forceps which pick up only the bleeding point, and suture materials of fine silk on atraumatic sharp needles. Only by great delicacy in handling tissues can the ensuing tissue reaction be reduced to a minimum. Repeated sponging is avoided and haemastasis is secured by tying bleeding points with fine catgut or silk. All deep spaces are obliterated by accurate approximation of the tissues in layers. The skin suture may be continuous or interrupted but each stitch must produce eversion of the edges, of the wound. It is
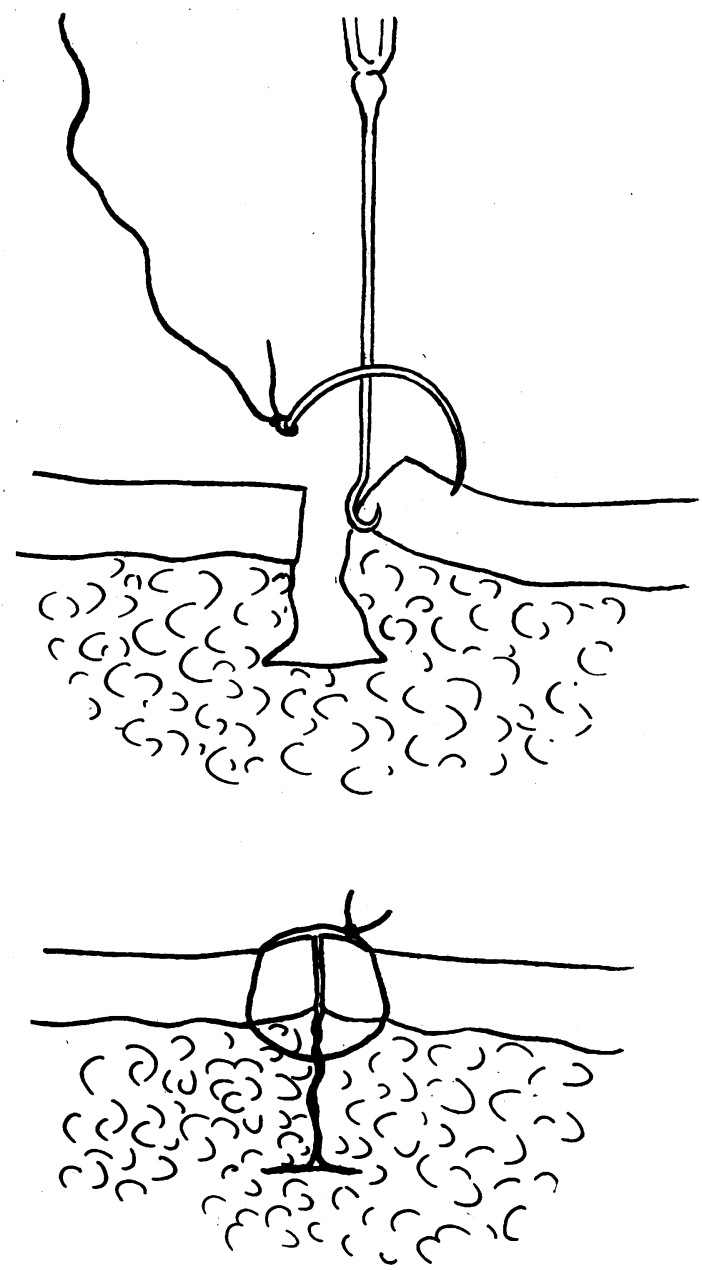

Fig. r.-Detail of stitching technique. 
important that the stitch should be just sufficiently tight to secure approximation of the wound. Too tight a stitch causes unnecessary stitch marks, and if the stitches are spaced too closely together the skin edges will be ischaemic and healing will be delayed.

The needle enters the skin at not more than $\frac{1}{8}$ in. from the edge and passes into the deeper tissues, securing a slightly greater ' bite' until the needle reappears $\frac{1}{8} \mathrm{in}$. from the other skin edge (Fig. I).

It is a cardinal rule in plastic surgery that closure of a skin defect must not be achieved under tension, because the resulting scar stretches and if it does not lie parallel to the normal skin creases hypertrophy or keloid formation may occur. Up to about the age of three, scars on the whole behave satisfactorily and rarely become keloidal. From three to 17 years of age scars are prone to be disappointing. Certain patients older than 20, particularly those with red hair or deeply pigmented skin, tend to produce very unsightly scars, especially if the direction of the scar crosses the normal skin creases.

\section{Closure of Small Skin Defects}

Removal of tissue, including skin, for example following excision of a rodent ulcer, may leave a defect that cannot be closed easily by direct suture that obeys plastic principles.

The closure of all small defects involves mobilization of the wound margins and 'advancing' the tissues to cover the defect. The size of defect that can be closed by this simple method depends on the elasticity of the skin, after undermining the skin for an area approximately five to nine times greater than the defect. There are areas in the body where loss of skin cannot be made good by simple 'advancement' flaps or extensive undermining and suture. An example is the lower limb, where the skin is relatively inelastic, and if it is stretched its viability is considerably impaired. Similarly defects situated near the eyelids, mouth and nose will cause distortion of the surrounding tissues if sutured under tension. This may lead to subsequent ectropion and exposure of the globe, or to asymmetry of the mouth and dribbling.

Most small defects of the skin can be resolved into three main geometrical figures, namely a circle, a triangle and a rectangle. An irregular defect can be resolved by paring the skin edges to form a combination of two or more of these figures. The surrounding skin must be widely undermined for a total area of between five and nine times the area of the defect. Fig. 2a, b, c, d illustrates the method of closing these defects.
Small puckers or dogears may be produced at the corners of the closed incision. This redundant skin is carefully excised so that the final incision is flat though slightly prolonged.

An important step in every operation is the mechanical approximation of the divided tissues to obliterate dead spaces and minimize the ultimate scar. If the skin is approximated and the deep tissues ignored, exudates and haematoma will accumulate in the remaining dead space and invite infection; this will delay healing and the surface scar, regardless of the care with which the skin wound may have been closed, will stretch because
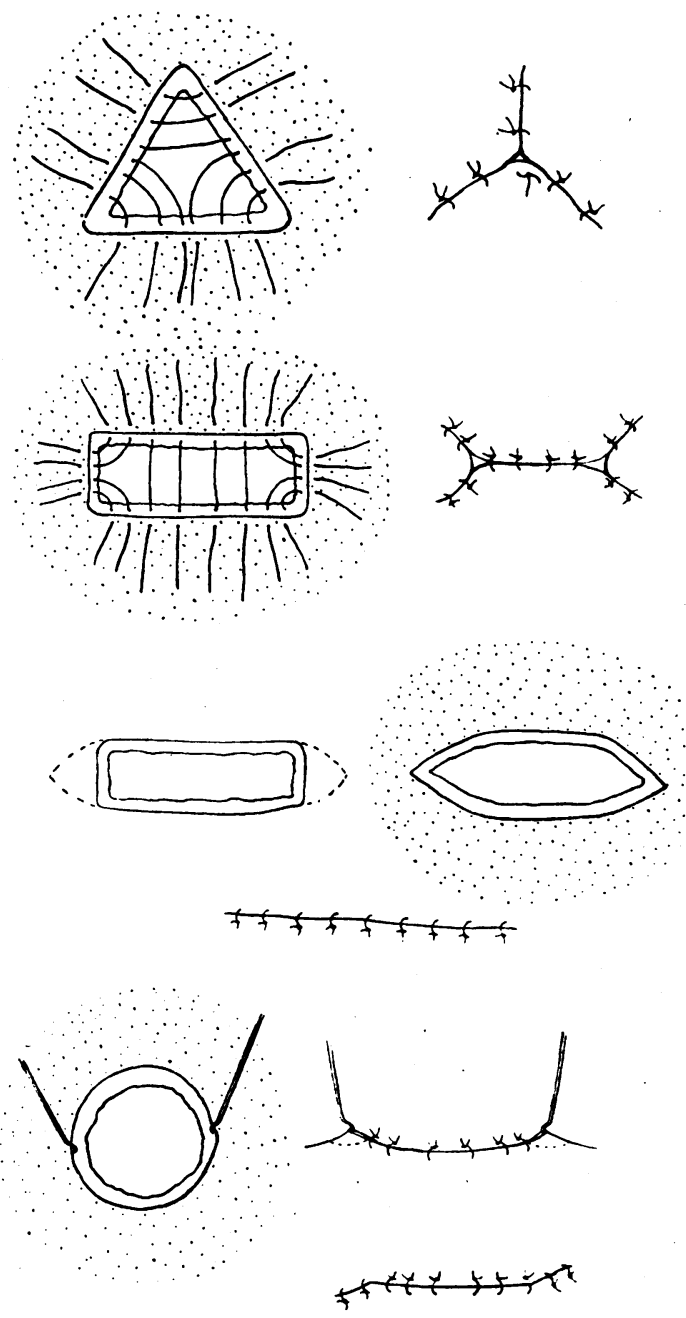

FIG. 2.-Methods of closure of defects (the stippled $\mathbb{E}$ areas indicate areas of undermining): (a) Triangular defect. (b) Rectangular defect. (c) Rectangular defect, alternative method with triangular excisions at each end. (d) Circular defect. Note final excision of dog ears. 
of insufficient support. In other words, the subcutaneous tissues must be carefully united in layers.

A good scar may be secured if:-

I. The skin is incised vertically and not at an angle.

2. The incision lies parallel to the normal ' grain' or natural creases of the skin.

3. The incision is of adequate length so that unnecessary retraction and tissue manipulation is avoided.

4. Haemostasis is absolute.

5. Dead spaces are obliterated.

6. Stitches are not inserted under tension.

7. Infection is avoided.

Stitches are only a method of fixation, bringing the two cut surfaces into the closest apposition until healing has begun. Early removal of stitches, particularly on exposed areas of the body, is important, as unsightly stitch marks will ruin an excellent scar. Pre-operative and post-operative irradiation of the skin may be necessary to secure the best result.

\section{Closure of Raw Areas with Skin Grafts}

In the surgery of trauma and, indeed, as a result of a planned surgical operation, a skin defect is not uncommon. Sometimes a wound may break down leaving a raw area which takes a long time to heal by secondary intention. Primary ' closure ' of large skin defects with skin grafts is a simple problem. Special training is unnecessary and little skill is required.

Skin grafts of most use to general surgeons fall into two categories:-

I. Pinch grafts.

2. Split skin grafts.

\section{Pinch Grafts}

The resurfacing of denuded areas by the use of small 'pinch' grafts is the oldest and simplest method of skin transplantation. It involves no special preparation, is easy of execution and can be done under local anaesthesia. The dressings and instruments are not elaborate and the after care is not irksome. These minute transplants will ' take' on any raw surface' and under conditions contraindicating the use of other more complicated methods; indeed, they will often survive even in the presence of considerable suppuration. Furthermore, should a portion of the grafts fail, it is no great calamity since the loss can easily be remedied by a repetition of the process.

Technique. The granulation tissue is prepared for grafting by the application of gauze soaked in normal saline and this is changed four-hourly. A light pressure dressing is applied. After three to four days pale, heaped up granulations with a heavy infection can be made to flatten out and become cherry red in colour, the surface resembling a well-cut lawn.

A word of warning is necessary. Local measures alone are not enough to prepare a granulating area for skin grafting if the haemoglobin is below 60 per cent. Blood transfusions must be given. In my experience a raw area is safe to graft when the skin edge is beginning to grow in or epithelialize. No mention has been made of the use of antibiotics to help sterilize the raw area. It is a controversial subject and it is largely a matter for personal preference, but I prefer to withhold the application of all antibiotics unless there is a special reason.

The grafts are obtained from an area of skin bordering the lesion. A small island of epidermis is raised on the point of a cutting needle, and with a sharp knife, such as a B.P. No. II, a cone of skin 4 to $5 \mathrm{~mm}$. in diameter is cut off and transferred to the granulating area, raw surface downwards. The graft consists of epidermis and part of the dermis (Fig. 3a, b). If the graft has been properly cut a slight ooze of blood takes place in the crater.

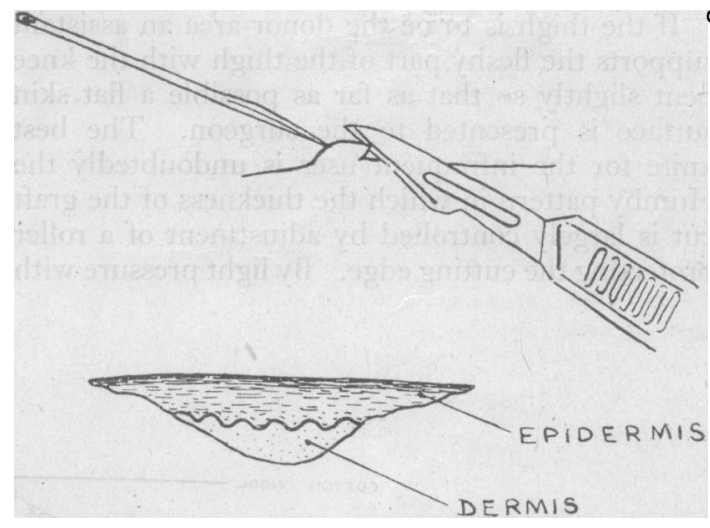

Fig. 3.-(a) Cutting a pinch graft. (b) Section of graft.

Care must be taken to avoid cutting these grafts at a deeper level, otherwise permanent scars will be left on the donor area and the thick layer of connective tissue on the base of the graft will interfere with its viability. The closer the grafts are placed the better, since the proliferation from their edges is limited, so the more numerous the grafts the more rapid the healing.

After the grafts have been placed a layer of tulle-gras is laid over the area. Above this are placed a few layers of gauze wrung out of saline 
solution. These in turn are covered by two or three layers of dry gauze, the whole being held in place by a bandage or adhesive plaster. If the area that was grafted was dirty a change of dressing is required every 24 to 48 hours, depending upon the amount of discharge. If the base was clean the dressing is left undisturbed until the part is completely epithelialized.

\section{Split Skin or Razor Grafts}

Application of grafts of this type is simple and rapid. The grafts may be obtained in any size, limited only by the available donor surface and by dexterity of the operator. Owing to their thinness and comparative avascularity they are capable of receiving nutrition through direct osmosis from the surrounding lymph spaces. The donor area heals quickly and spontaneously without serious scarring. The grafts are subject to discoloration which may render their use objectionable on exposed parts, particularly on the face.

Technique. The donor area is preparea and draped in the normal manner.

The cutting of the graft requires no great skill provided a flat surface is presented to a knife that is really sharp. Even in the hands of experts the cutting of a graft can be an impossibility with a blunt knife.

If the thigh is to be the donor area an assistant supports the fleshy part of the thigh with the knee bent slightly so that as far as possible a flat skin surface is presented to the surgeon. The best knife for the infrequent user is undoubtedly the Humby pattern in which the thickness of the graft cut is largely controlled by adjustment of a roller protecting the cutting edge. By light pressure with

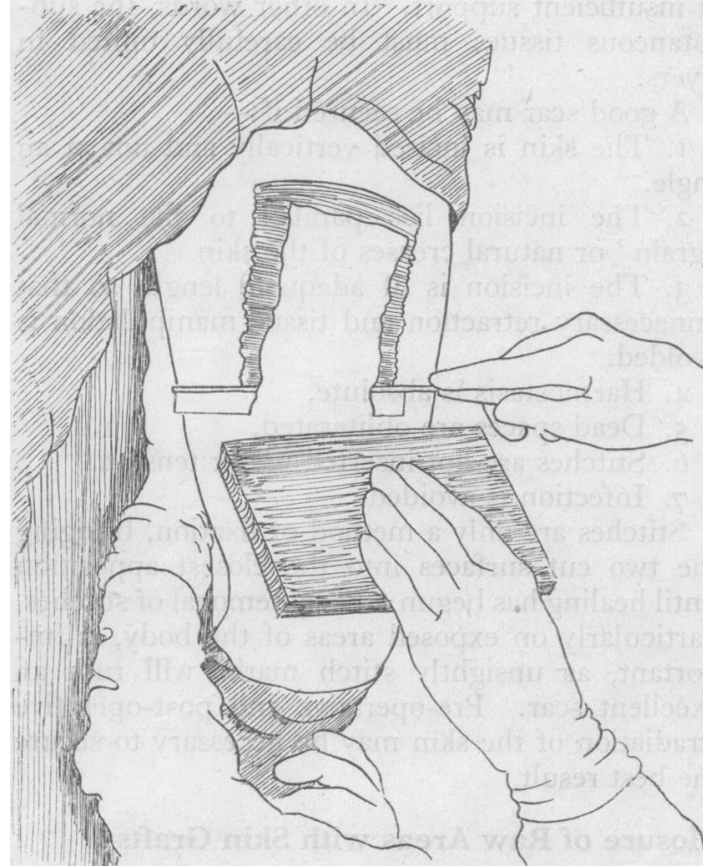

FIg. 4.-Cutting a Thiersch graft from the thigh.

a flat board placed parallel to the cutting edge of the knife, the skin surface is tightened and flattened. By smooth cutting movements rather like slicing ham, a graft may be cut keeping the graft board about $\frac{1}{2}$ in. to $I$ in. in front of the moving knife edg e (Fig. 4).

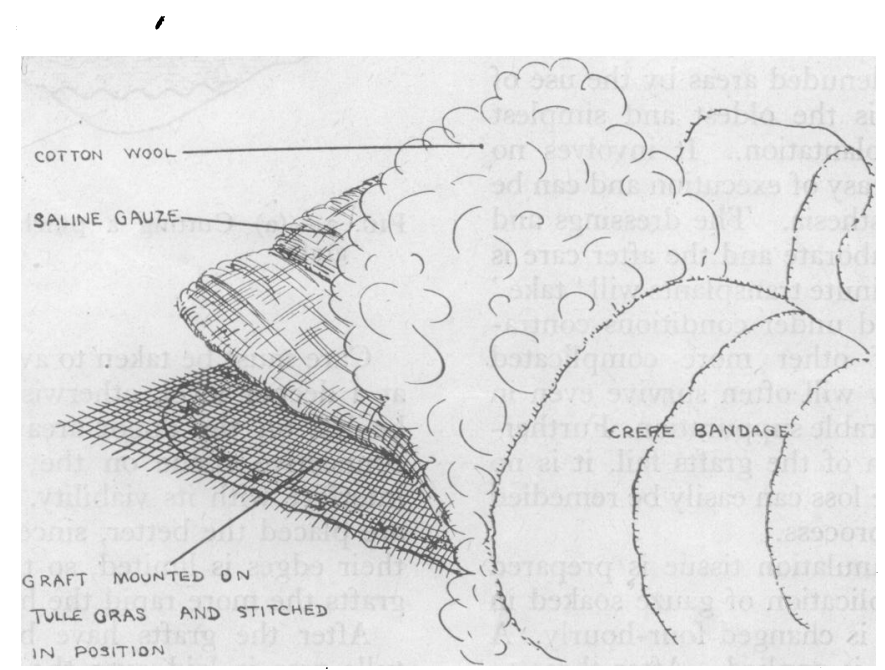

Fig. 5.-Dressing for free graft. 
The split skin graft is spread on to tulle-gras with the raw surface outwards, and transferred to the recipient area. The whole is secured by stitching the graft and tulle gras to the skin around the defect (Fig. 5). The after care and dressings have already been described and are the same as that given for pinch grafts.

The donor area is covered with tulle-gras dressings and liberally protected with cotton wool; the dressings are secured with a sterile bandage.

I do not think flaps and tubed pedicle grafts come within the scope of this subject. Some general surgeons have had experience of their application, and the following remarks do not apply to them. The results following the use of tubed pedicles and flaps is often very disappointing to the surgeon and may be disastrous in inexperienced hands. It takes considerable training and experience for the plastic surgeon to obtain a sound working knowledge of what to do and what not to do. For instance, the Imre flap used on the face to repair defects situated around the lower eyelid appears relatively simple. So it is, if the tip of the flap survives (and it is easily lost), if haemostasis is perfect (and it is difficult to secure), if the stitching is not so tight as to cause a necrosis of the skin edge, if the flap is designed big enough, if the incisions lie in the same direction as normal skin creases and, finally, if the subject is old and has an abundance of loose skin that can be mobilized to replace the defect. The scales are weighed heavily against the surgeon who, without previous training, attempts what may appear to him a simple procedure.
However, a scalp defect is frequently encountered, and it is often necessary to move the scalp by carefully designed flaps to cover a dural or bony defect.

\section{Special Areas \\ Scalp}

The scalp is almost completely inelastic, and this feature governs any plastic repair in this area. Very small defects are often impossible to close by direct suture. Neither will extensive undermining of the adjacent scalp allow the tissues to be advanced over the defect. The use of advancement 'flaps' depends on the elasticity of the tissue; because the scalp will not stretch defects in this area must be closed by flaps. The abundant more complex blood supply of the scalp makes the use of very large flaps a relatively safe procedure. The flap should be designed with its base towards the main blood supply of the scalp and its size should be four or five times greater than the defect. The greatest and most frequent mistake is to design too small a flap so that the defect is incompletely covered.

The scalp flap is elevated completely, leaving pericranium intact. It is then rotated and sewn into its new bed. It is possible that a 'dog ear' will form when the scalp flap is moved into its new position. This is of no importance at this stage, the object of raising a flap being to cover a specified defect. 'Dog ears' are easily trimmed out at a later date. There is obviously a raw area or secondary defect from the site where the scalp flap previously lay. This is covered by a split

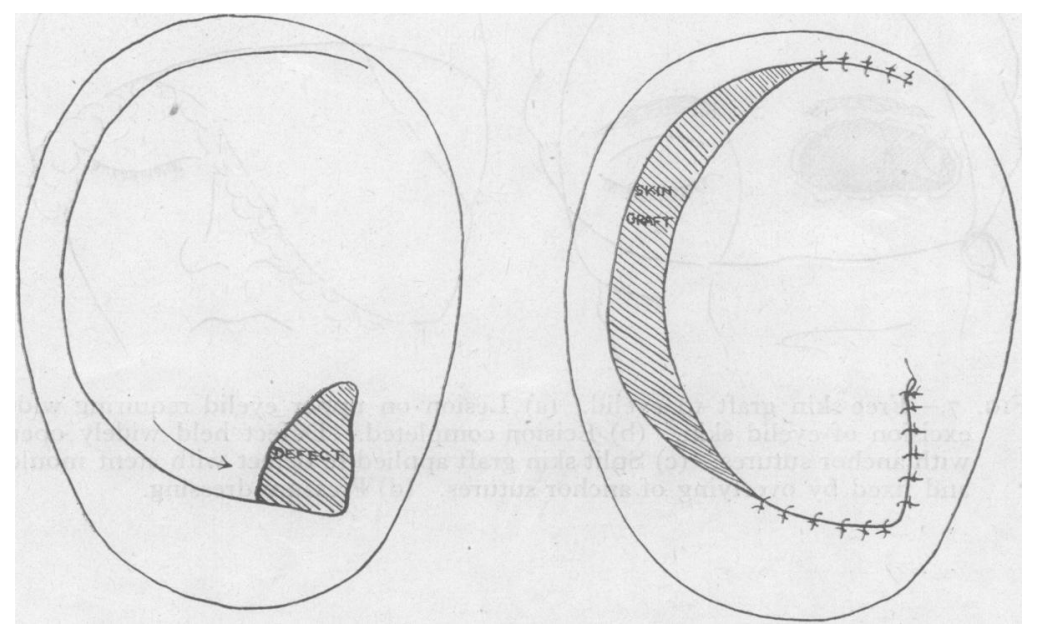

FIG. 6.-Closure of scalp defect with rotation flap. Skin graft to secondary defect. 


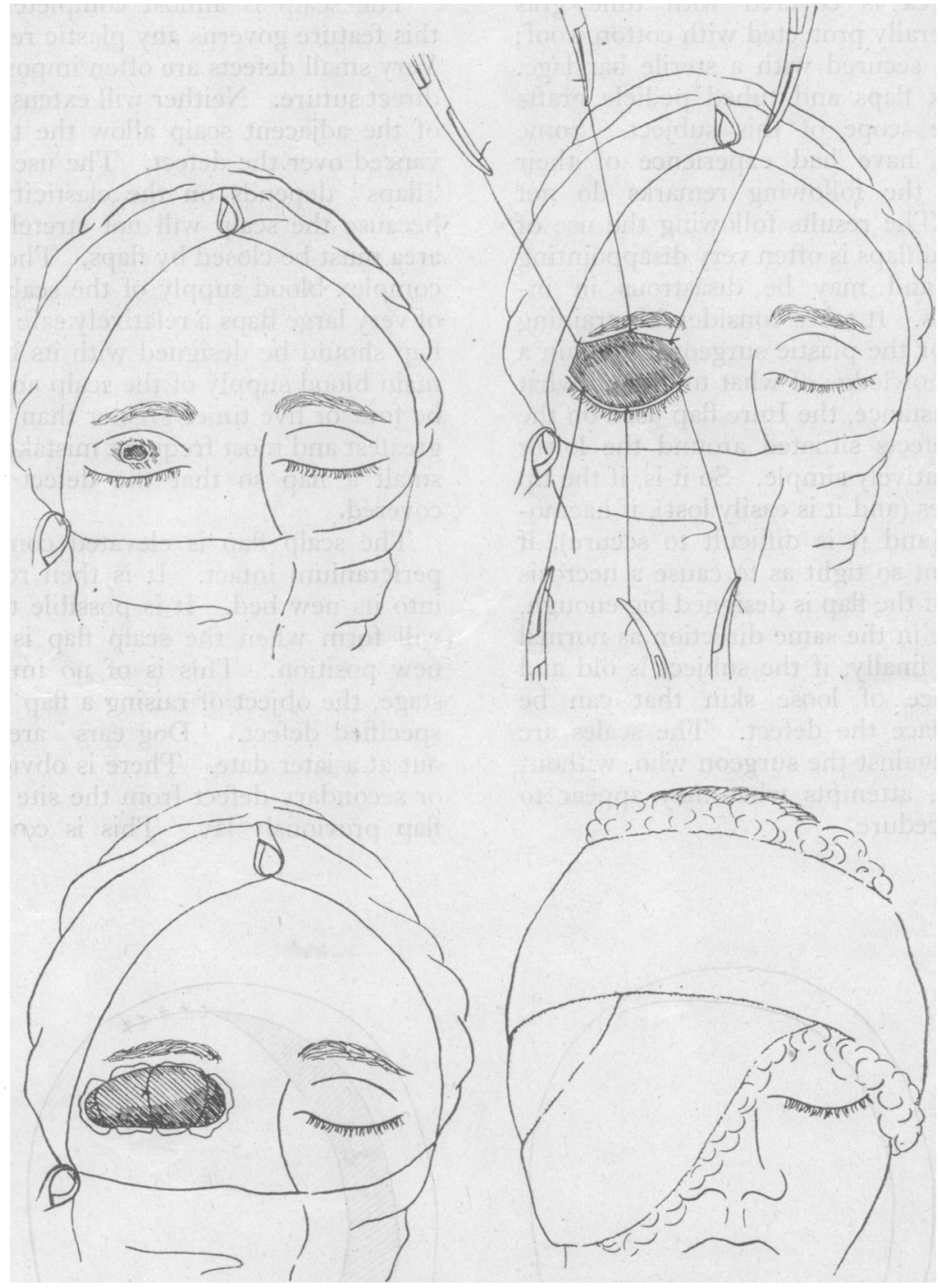

Fig. 7.-Free skin graft to eyelid. (a) Lesion on upper eyelid requiring wide excision of eyelid skin. (b) Ecision completed. Defect held widely open with anchor sutures. (c) Split skin graft applied to defect with stent mould and fixed by overtying of anchor sutures. (d) Pressure dressing. 
skin graft secured with a few interrupted fine stitches (Fig. 6). As an emergency measure it is justifiable to use hairy scalp flaps to close forehead defects involving bony loss. The hairy scalp can subsequently be returned to its original position and repair of the forehead defect achieved by a secondary free graft.

Face

The surgery of the face presents special problems since the result is visible to all. A clean laceration inflicted with a sharp knife can be converted into a hideous scar by the surgeon who closes the wound in a clumsy fashion. If skin has been lost in an accident or necessarily removed in the excision of malignant disease, closure of the defect is achieved by adding tissue from the vicinity or covering the raw area by a free graft. The use of adjacent tissues implies closure by local flaps. Their use requires special training and they cannot be described satisfactorily in a short paper.

A defect that is too large to be covered by simple methods is best closed by the application of a split skin graft. The surrounding tissues are thereby not distorted and the chaotic sequelae of tight strangling sutures are eliminated, so that in due course the surgeon will face a problem unobscured by avoidable scarring and distortion of tissues. There are many defects on the face which it is not proposed to discuss, because I consider them entirely within the province of the plastic surgeon. Lesions of the upper or lower lips not exceeding one-third of the width can readily be closed by excising a complete $\mathrm{V}$ section of the lip including skin, muscle and mucous membrane. The raw areas are united in layers securing accurate union of mucous membrane, muscle and skin. The cosmetic effect is satisfactory. More complicated repairs involving the Abbe or Estlander flap or the Gillies 'fan flap ' require the full plastic armamentarium for their satisfactory conclusion.

Lacerations of the eyelids, particularly those involving the lash edge, present a special problem. The multitudinous methods of repair, each with its enthusiastic advocate, testifies to the difficulty in obtaining a satisfactory result. Scars lying vertical to the lash edge tend to contract giving a notched deformity. Full thickness defects of the eyelids present special technical problems which is outside the scope of this article.

Skin losses of the eyelids which are larger than can be closed by some obvious simple method are best covered by a thin split graft. The skin graft is most satisfactorily applied to the raw area on a stent mould. A small piece of stent of suitable size is rendered soft by immersion in hot water. When workable it is moulded to the shape of a sausage and applied to the defect and cooled with cold water. It is then dried and a split skin graft of suitable size is wrapped on to the mould, raw surface outwards. The mould with its skin covering is then secured to the raw area by stitches inserted into the cut skin edge, the long ends of which are tied over the mould. A light pressure dressing is applied and the dressing left undisturbed for three to five days. Subsequent dressings are carried out as for any other free graft (Fig. $7 a, b, c, d$ ).

Neck

The most important plastic procedure in the neck which comes within the scope of the general surgeon is the technique for breaking a vertical scar which has contracted and is limiting movements of the neck. This is the Z-plasty and it is of use only if the scar is lineal and tends to produce a web. The surrounding tissue must be sufficiently vascular to withstand the procedure. It is carried out as follows. With the scar under tension, the proposed incisions are marked out. The longest line of the $Z$ is the scar itself. The arms of the $\mathbf{Z}$ begin at each end of the scar and are marked out at an angle of $60^{\circ}$ to the scar and about two-thirds of its length (Fig. 8). The incisions are made following the pattern, and the two broad based triangular flaps thus formed are undercut and fully mobilized. The position of
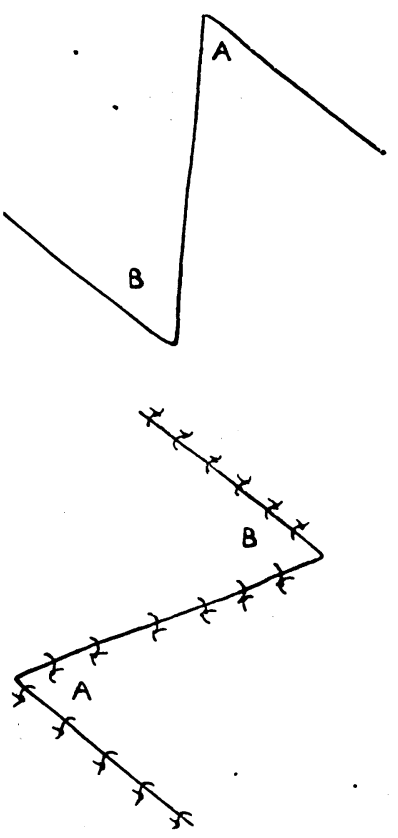

FIG. 8.-The $Z_{i-j \text { lasty }}$ 
the flaps is then transposed so that the tip of one flap is sutured at the opposite side of the base of the other flap. The $\mathrm{Z}$-shaped incision is sutured, but the long line of the scar now lies transversely across the original scar pull.

\section{Chest}

Skin defects of the chest wall sometimes arise during resection of a carcinoma of the breast. Complicated flaps are not practical and the defect should be closed in the simplest manner. The incision is sutured as far as possible and the remaining area is covered with a split skin graft. Primary healing is secured and the donor area is healed within ten days. The technique for cutting the graft and subsequent dressings have been described. cision is indicated when the previous incision was़్ paramedian in type.

Relaxation incisions if used at all should be closed by the application of a skin graft. The extra? time taken to do this is rewarded by healing. obtained in the shortest time.

\section{Lower Limb}

Probably the commonest problem in the lege which besets the general surgeon is the treatment of varicose ulcers. The following remarks are not intended to cover the entire subject but to describe some methods of use in this controversial field $\vec{O}$ I am fully aware that some surgeons consider that skin grafts have a very limited use and the ulces may recur in spite of ' new skin.'

Varicose ulcers are seen in all stages of chronicity

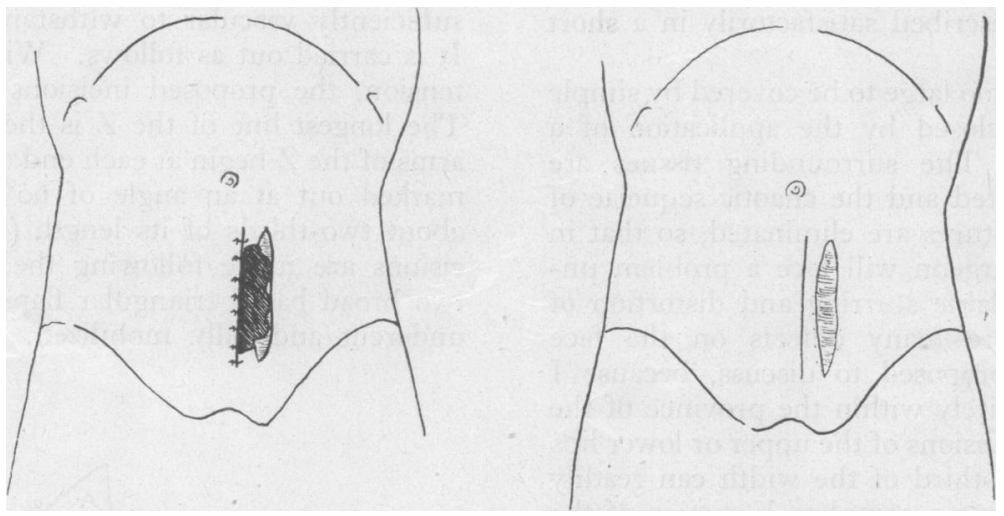

Fic. 9.-Diagram illustrating death of skin between two longitudinal incisions.

\section{Abdomen}

Great care is rightly given to closure of the abdomen following laparotomy, but a number of patients subsequently develop ventral herniae. This is more common in patients who have been subjected to more than one laparotomy. Some surgeons advocate that the abdomen should be reopened through an incision parallel to the previous incision, but not through the same incision if the same exposure is required as at the previous operation. This practice may lead to sloughing of the skin of the abdominal wall between the incisions and is associated with a very high incidence of hernia (Fig. 9). The old scar should be excised and the wound extended into normal tissue and the skin undermined extensively until normal rectus sheath is found. The peritoneal cavity is then opened through normal tissue. If speed is the sole consideration a transverse in- and sizes, with or without visible varicose veins Oedema of the foot and leg may be increased by local infection. Many patients do not seek relief until pain or profuse offensive discharge from the ulcer denies them sleep or the company of theis fellow men. Skin grafts have no place in the un? treated varicose ulcer. The varicose veins must beo ligated or injected, the superadded infection and: oedema must be controlled. A lengthy period ins bed may be indicated according to the severity of the ulcer, and it may take several weeks to achieve्w a very slowly healing ulcer. Recent ulcers with little surrounding fibrosis and with a soft red base can be successfully healed by pinch grafting the raw area. This can sometimes be accomplished on an out-patient. The larger more indurated ulcer with extensive scarring in the base and surroundingo tissues is a different problem. Even if healing is obtained by the application of pinch or split skint 


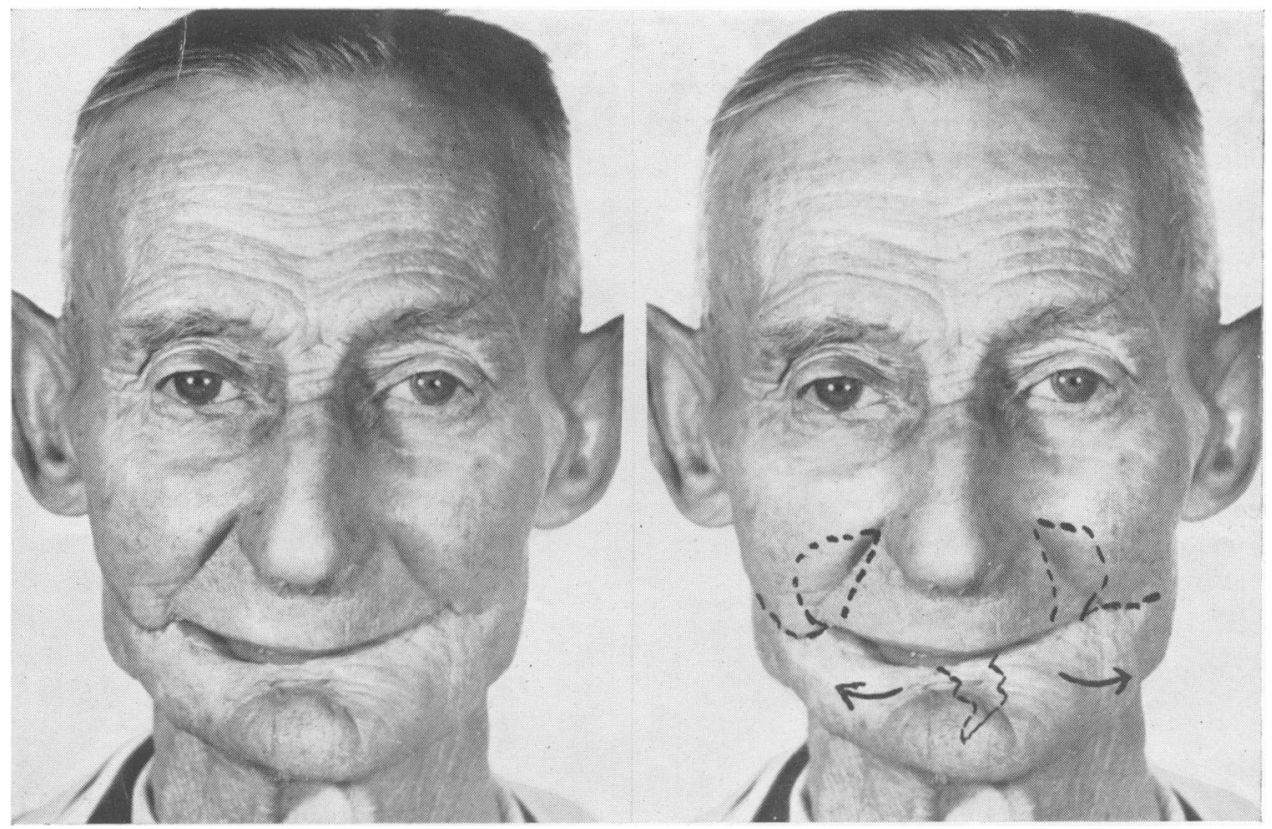

Fig. 10.-The whole lower lip was resected for an epithelioma. Both cheeks were extensively mobilized and an attempt made to secure union in the midline under tension. The result was catastrophic. The wound broke down and a fistula formed in the midline. The new 'lower lip' was totally inadequate and was stretched as a tight band beneath the upper lip. Dribbling was constant, mastication and phonation were difficult. A repair was performed in which the previous 'flaps' were returned to their normal position and tissue was used where it could be spared, that is from the nasio-labial region. The dotted lines indicate the method of reconstruction.

grafts, the healed ulcer surface remains unstable and easily breaks down. A wide excision of the ulcer and surrounding scar tissues is indicated, the resulting raw area being covered with a split skin graft. Even after healing, the ulcer diathesis remains and the limb must be permanently supported by elastic bandages or stockings, applied before getting out of bed. The ulcerated leg with a severe periostitis and endarteritis is in most cases unsuitable for the methods described above, and the services of a plastic surgeon should be sought to consider the possibilities of a cross leg flap.

The general surgeon, having read so far, will feel that I have allowed him a very limited field in plastic surgery. If Maingot's predictions are right and an atraumatic technique is adopted for general surgical procedures, a much greater field may become available. The following cases are discussed to impress upon all surgeons ambitious to do reconstructive work that an atraumatic technique is essential.

FIG. I 1.-The longitudinal contracted scars crossing the metacarpo phalangeal joint creases have resulted in limitation of extension. The patient stated that the joints of his hand had a wider range of movement before the operation for removal of the palmar fascia for Dupuytren's contracture.

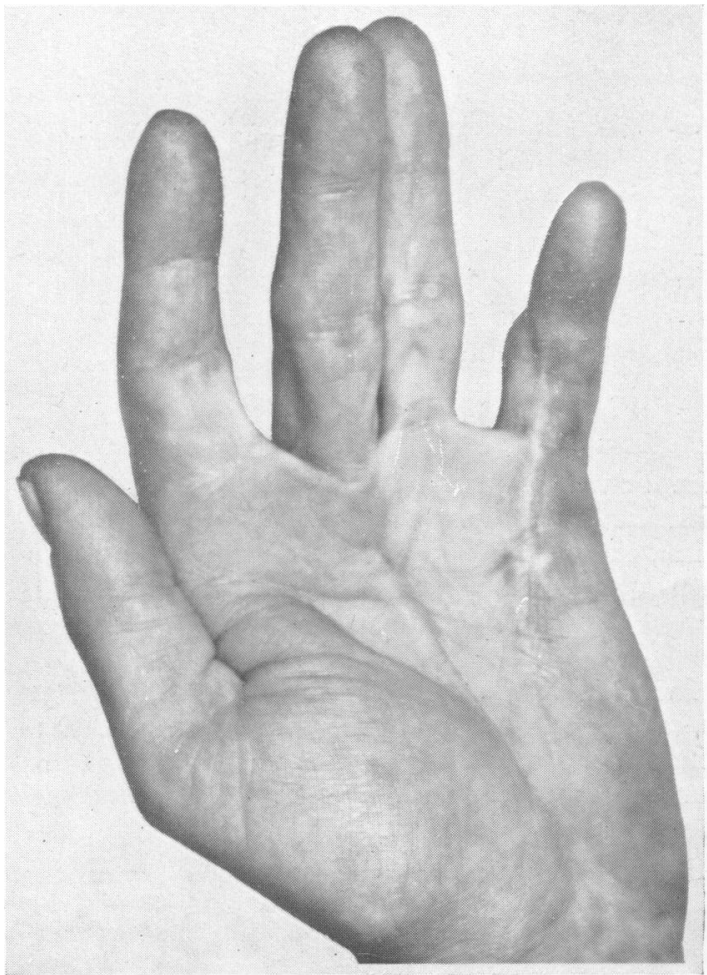




\section{Case I (Fig. 10)}

Large resections for cancer of the mouth must not be repaired by any method of which the surgeon has had no previous experience. Wide resection of the growth, yes, but attempted reconstruction of the oral sphincter without technical equipment or knowledge, no.

If the wound does not break down following illconsidered repair the unfortunate sufferer may have a new disease. If the cheeks had been widely used in the repair, mastication may be impossible and dribbling a daily nuisance. Many old ture is difficult enough without vitiating a possibly good result by ill-placed incisions.

\section{Case 3 (Fig. 12)}

Frequently the general surgeon may be tempted to repair a skin defect by local flaps or a tubed pedicle flap. It is not enough that the tubed pedicle flap is eventually transplanted to the recipient area; the point is that the tubed pedicle flap should be really necessary and that it should merge with its surroundings. Repair must have function as its main aim for if this is not achieved

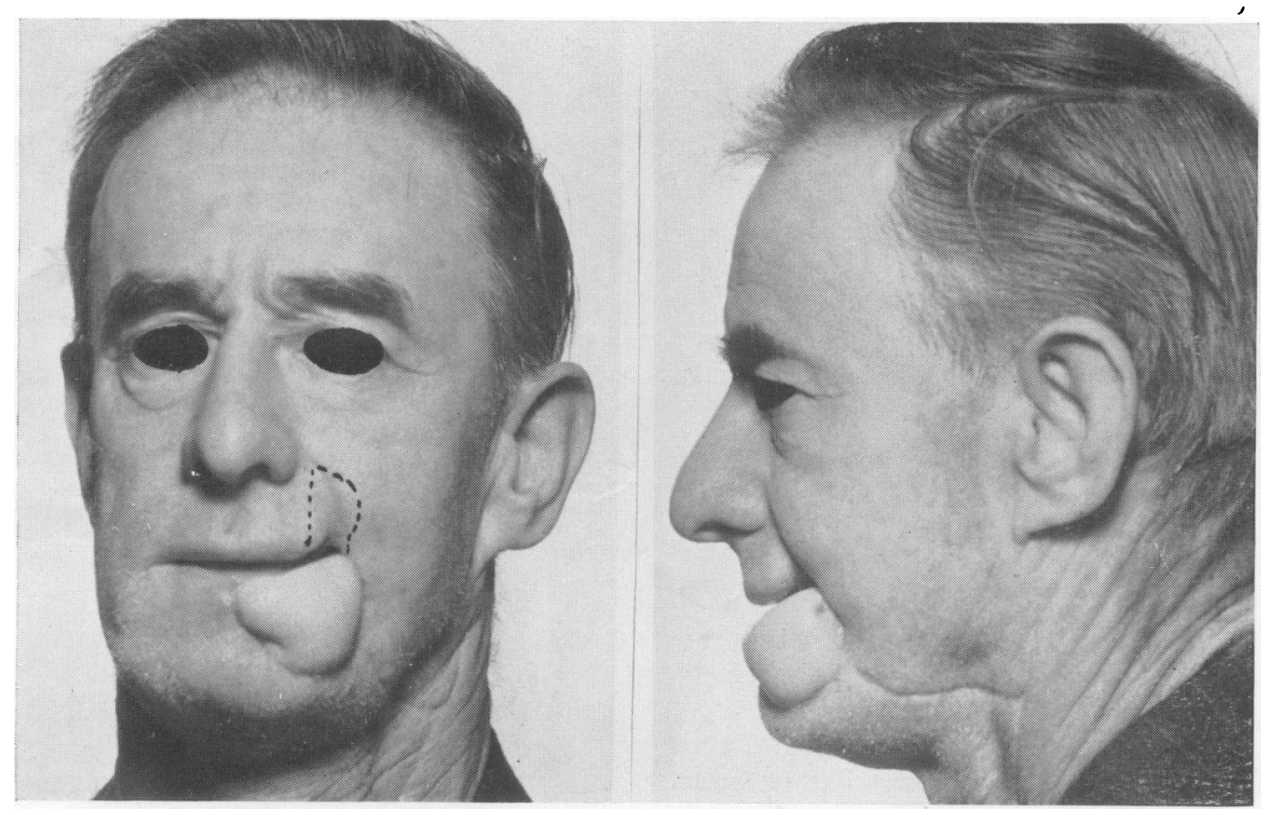

FIG. 12.-This man lost a small full thickness section of his lower lip in an automobile accident. A repair was carried out by a tubed pedicle flap. The flap remained unsightly, anaesthetic and the man was troubled with persistent dribbling. A repair of the Estlander type (shown in dotted outline) would have given a better result, with retained sensation and, therefore, without dribbling. The cosmetic result would also have been better.

men for whom this type of operation is performed wish for little more than to be able to smoke, drink, eat, talk and sleep. If their oral sphincter is destroyed their last days will be a misery.

\section{Case 2 (Fig. II)}

Incisions in the palm of the hand should never be made longitudinally across a joint crease. These incisions may heal with contraction of the scar which prevents full extension of the involved joints. The worst examples are to be seen following unwise incisions for acute infection of the hand or for Dupuytren's contracture. They are inexcusable. The surgery of Dupuytren's contrac- on account of an ill-judgud type of repair, the series of operations will have been pointless.

The three case photographs which follow are shown to illustrate problems that can only be solved by ' plastic' surgery.

\section{Case 4}

This woman had an extensive basal celled carcinoma of her forehead involving the left eyelids, left ear and extending across the nose (Fig. 13). The whole mass was excised including much of the left frontal bone, the left eye and part of the left ear. The defect was covered by a large hairy scalp flap. One month later, the scalp flap 


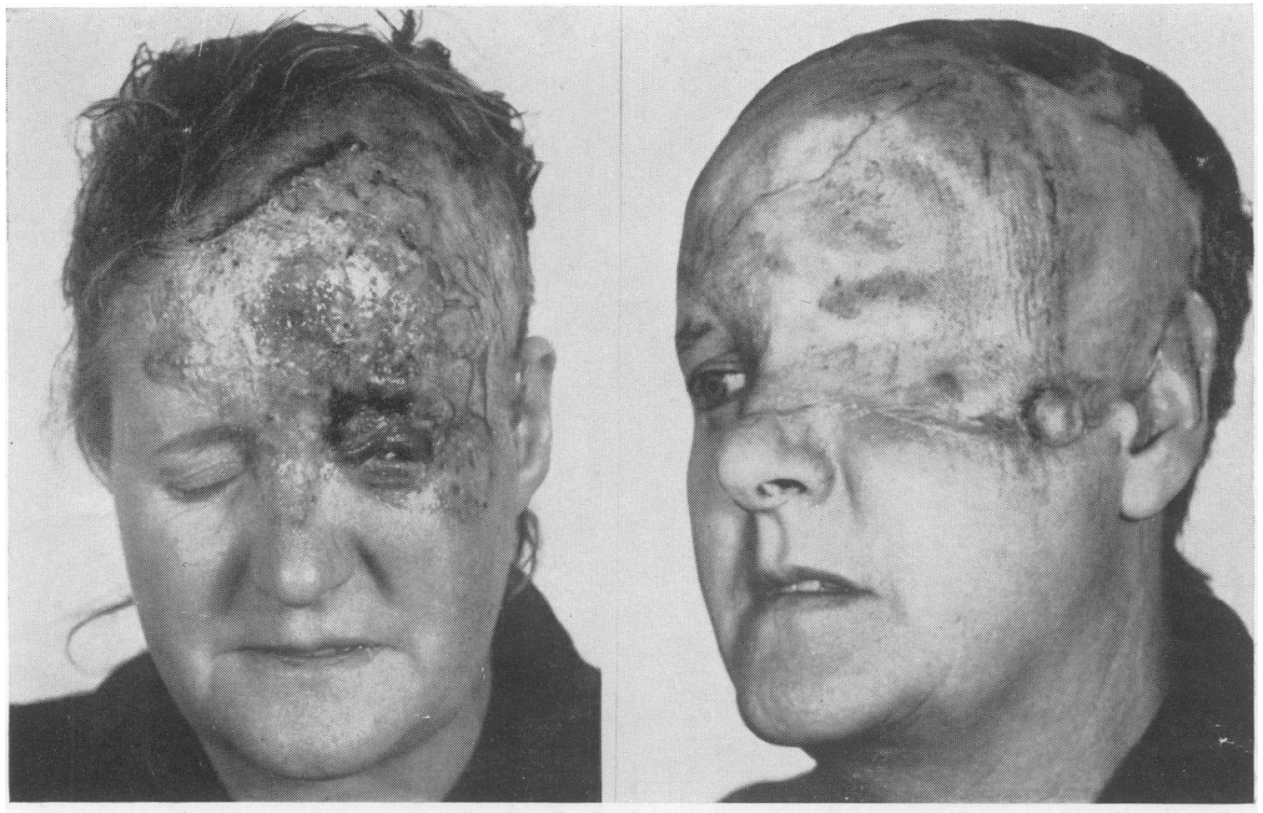

Fig. 13.-Extensive basal-celled carcinoma of forehead, before and after treatment.
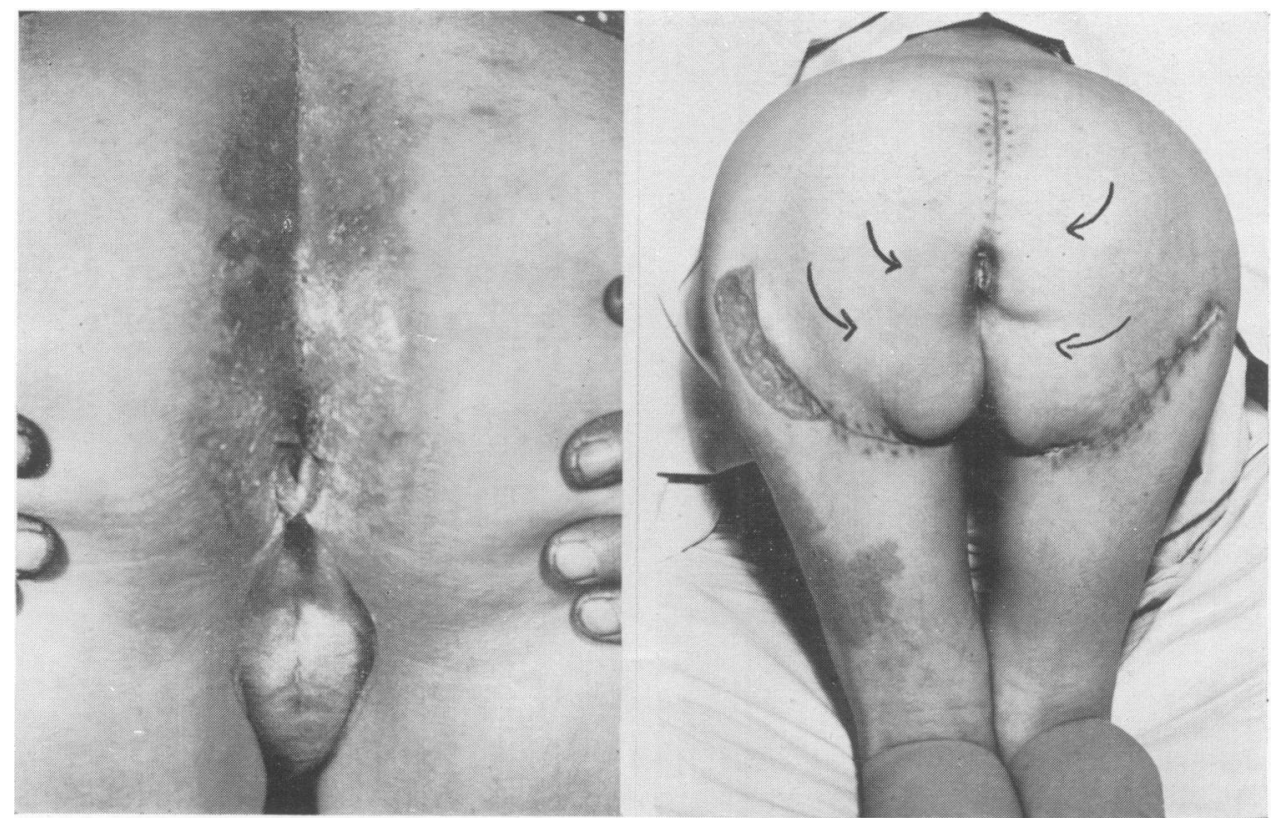

FIG. 14. - The relief of pruritus vulvae by radiation has, in some cases, resulted in a radiation dermatitis. This must be treated by excision which gives immediate and lasting relief. The defect resulting from excision of all affected tissues is satisfactorily closed by using two large rotation flaps. 


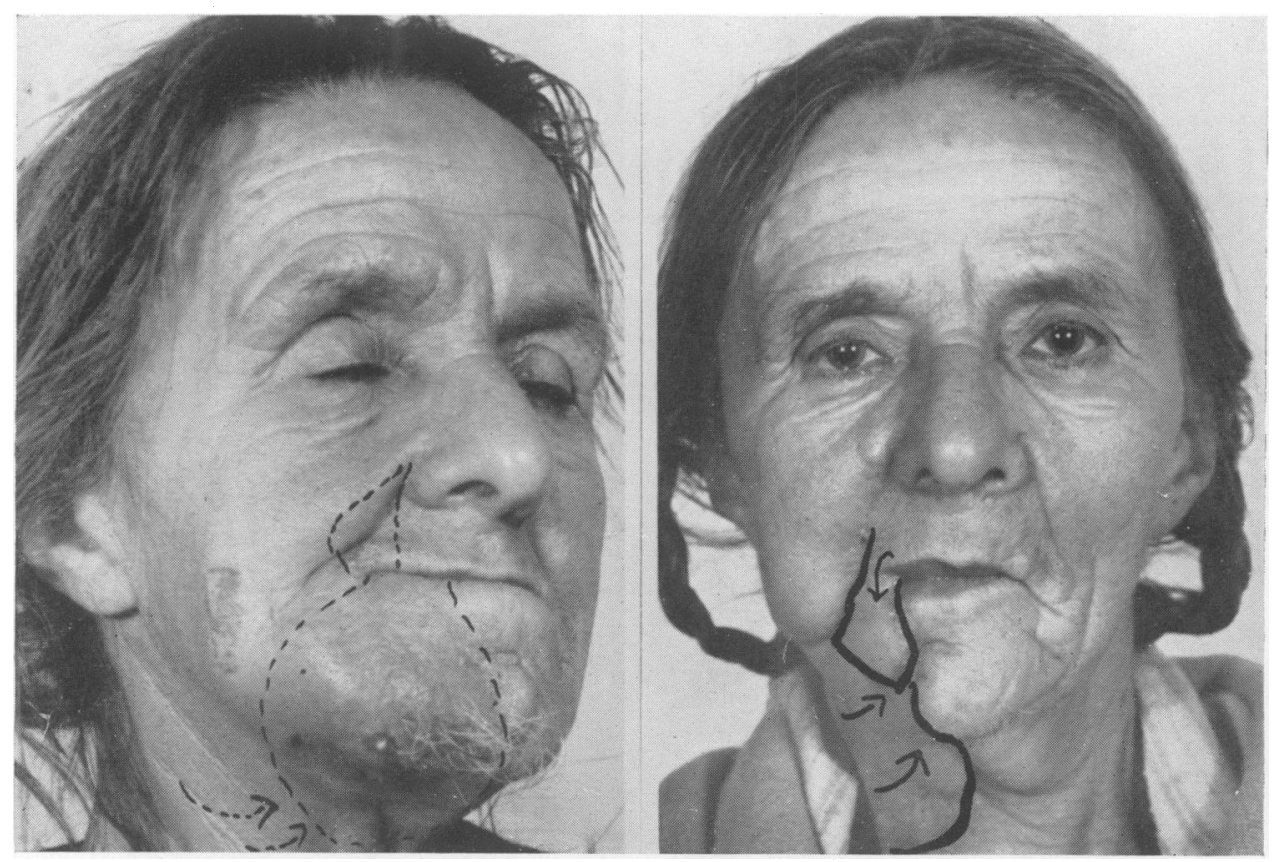

Fig. I 5.-(a) An attempt had been made to remove the growth by the intra-oral route three months previously. A large malignant ulcer was present on the alveolar ridge, the base invading the skin of the submental region. (b) The patient on discharge, I4 days after wide excision of the growth and immediate repair. Function of the mouth was excellent and the result cosmetically acceptable. As this patient had not worn dentures for several years mastication with the remaining half mandible was satisfactory.

was returned to its normal position and the bed it had occupied was closed by grafting the raw area with a split skin graft. This two-stage operation was necessary to allow the orbit to fill up and acquire a suitable bed for skin grafting.

\section{Case 5 (Fig. 14)}

The widespread use of radium and X-rays as therapeutic agents has resulted in a considerable number of cases of radiation dermatitis. Many of the minor burns heal and remain healed, but the ulcerative type, once established, demands complete excision. Pain, itching, ulceration and discharge combine to make the sufferer's life unendurable. Deformity from contraction is sometimes present. If the burn is on the face the unpleasant and disfiguring appearance is sufficient to make the patient seek relief. Lastly the real possibility of epitheliomatous change cannot ever he excluded, and for this reason total excision is indicated.

\section{Case 6 (Fig. I $5 \mathrm{a}, \mathrm{b}$ )}

This unfortunate patient, aged 67 , was referred to the Plastic Unit, East Grinstead, suffering from an intra-oral carcinoma ulcerating through the tissues of the chin on to the skin. It was grossly? infected. Wide excision of the tumour mass, in $=0$ cluding half the mandible, left a large defect Immediate repair was achieved using a full thickness ' $f$ an' flap to reconstruct the corner of the mouth and lower lip. A neck flap which includedo platysma closed the remaining sub-mandibular defect. This patient was able to return to hers home I4 days after operation with the slight dis-D ability that is associated with hemi-resection of the mandible - the remaining half of the mandibles swinging towards the side of injury when the mouth was opened.

\section{Acknowledgments}

I wish to thank Mr. Gordon Clemetson for the photographs, Mr. Robin Dale, F.R.C.S.E., for his? diagrams, Mr. C. R. McLaughlin, F.R.C.S.E., ando colleagues at East Grinstead for the considerableo help they have given me in the preparation of this article. 